\title{
La chambre cutanée : une fenêtre ouverte sur I'inflammation
}

La méthode des chambres cutanées, simple et atraumatique, permet d'analyser les différents composants de la réaction inflammatoire à divers agents (allergènes ou intermédiaires chimiques de l'inflammation), chez les sujets normaux ou malades.

Laurence Michel

Ingénieur biochimiste INSA, étudiante en $3^{e}$ cycle

\section{Louis Dubertret}

Professeur à la Faculté, directeur de l'unité U.312 de l'Inserm

\section{ADRESSE}

L. Michel, L. Dubertret : Inserm U.312, laboratoire de dermatologie, hôpital Henri-Mondor, 94010 Créteil, France.

\section{TIRÉS A PART}

L. Dubertret: Inserm U.312, laboratoire de dermatologie, hôpital Henri-Mondor, 94010 Créteil, France.

$m / s n^{\circ} 2$ vol. 4, féurier 88 a plupart des fonctions cutanées contribuent à nous protéger de l'environnement extérieur.

Par exemple, en tant que frontière naturelle, la peau s'oppose à la pénétration des produits chimiques et des bactéries et retient les fluides de l'organisme ; sa pigmentation nous protège contre la lumière. Cependant, le rôle protecteur de la peau implique non seulement une protection passive mais aussi la mise en œuvre d'un processus actif contre les agressions: l'inflammation, dont la persistance anormale, liée soit à la nature de l'agresseur, soit à une réponse mal contrôlée, peut aboutir à différents phénomènes pathologiques.

La capacité de la peau 5 à développer des réactions inflammatoires est très largement utilisée en médecine. La pratique des vaccins en est un exemple et les injections intra-dermiques, à visée diagnostique, un autre. Les processus inflammatoires qui s'expriment au niveau de la peau sont très variés et peuvent être le reflet de nombreuses maladies systémiques et métaboliques.

C'est dire à quel point il est tentant, pour étudier la physiopathologie et le traitement des processus inflammatoires, d'utiliser la peau humaine comme support expérimental vivant où viennent s'exprimer les capacités réactionnelles de l'organisme, qu'elles soient normales, pathologiques ou modifiées par des médicaments.

\section{Les fenêtres cutariées}

La couche cornée est imperméable et constamment renouvelée par la croissance et la différenciation épidermique: elle gêne l'étude des réactions cutanées, il faut donc l'éliminer. C'est ce que Rebuck a réalisé, il y a plusieurs dizaines d'années, en grattant l'épiderme avec un scalpel [1]. Il étudiait alors la réaction à un corps étranger par l'application de lames de verre sur la surface dégagée. Plus récemment, Senn et Jungi érodaient la peau avec une petite fraise utilisée en dentisterie et recouvraient la zone érodée d'une chambre plastique dans laquelle du sérum était introduit comme milieu chimiotactique [2]. Le débit de migration des polynucléaires attirés par le sérum hors des capillaires du derme dans la chambre cutanée pouvait ainsi être mesuré [2-4]. Toutefois, cette méthode était d'une reproductibilité médiocre du fait d'une certaine difficulté à définir la surface et la profondeur de l'abrasion. Elle pouvait être douloureuse et laissait parfois des cicatrices. Cette méthode a cependant permis de mettre en évidence une diminution de la migration des polynucléaires neutrophiles in vivo au cours de certaines maladies comme le diabète, la sarcoïdose et l'atopie. 
Figure 1. La technique des bulles de succion. Une capsule en acier (cliché A) est appliquée sur l'avant-bras, préalablement nettoyé à l'alcool, d'un sujet volontaire. Cette capsule possède à la base un trou de diamètre bien défini (4, 8,11 ou $14 \mathrm{~mm}$ ) et se trouve reliée par une tubulure fine à un manomètre associé à une pompe à vide. Une dépression légère de 200 à $300 \mathrm{~mm}$ de mercure peut être ainsi appliquée à la surface de la peau. En 2 heures environ. durée qui varie pour chaque volontaire, le fluide interstitiel cutané s'accumule entre le derme et l'épiderme, qui se décolle alors progressivement. Ce processus entraîne la formation d'une bulle, dite bulle de succion (cliché B). Histologiquement (cliché C), le décollement dermo-épidermique se fait avec précision et reproductibilité au niveau de la lamina lucida dans la membrane basale qui marque la frontière entre l'épiderme et le derme sous-jacent.
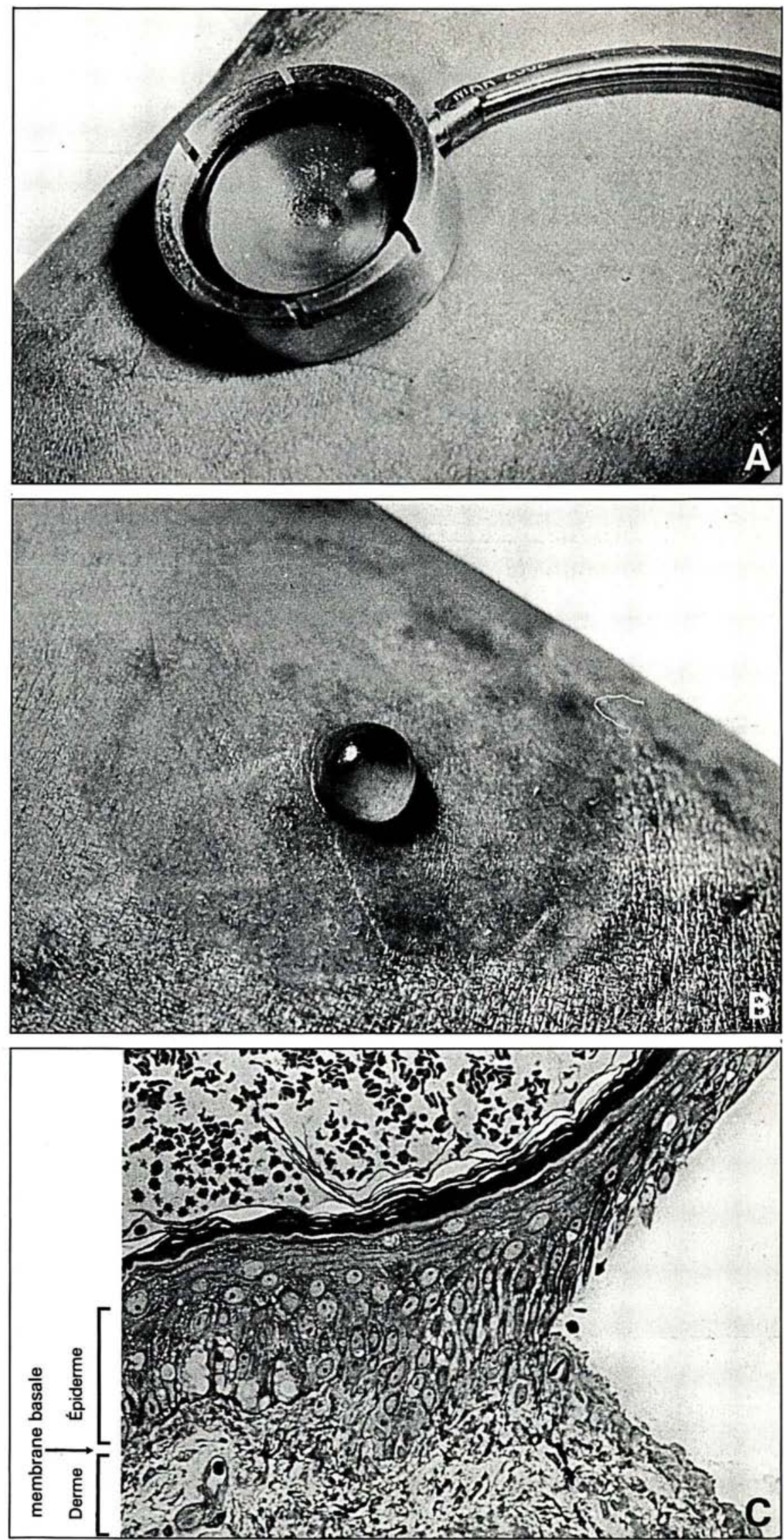

$\mathrm{m} / \mathrm{s} n^{\circ} 2$ vol. 4 , féurier 88

8. Michel L, Mencia-Huerta JM, Benveniste
J, Dubertret L. Biologic properties of LTB $_{4}$
and paf-acether in vivo in human skin. J
Invest Dermatol $1987 ; 88: 675-81$.

8. Michel L, Mencia-Huerta JM, Benveniste
J, Dubertret L. Biologic properties of LTB $_{4}$
and paf-acether in vivo in human skin. J
Invest Dermatol $1987 ; 88: 675-81$.

8. Michel L, Mencia-Huerta JM, Benveniste
J, Dubertret L. Biologic properties of LTB
and paf-acether in vivo in human skin.
Invest Dermatol $1987 ; 88: 675-81$.

1. Rebuck JW, Crowley JH. A method of studying leukocyte functioning in vivo. Ann

2. Senn HJ, Jungi WF: Neutrophil migration in health and disease. Semin Hematol

3. Norris DA, Lipman SH, Weston WL. Human monocyte chemotaxis: a quantitative in vivo technique. $J$ Invest Dermatol $1979 ; 72: 81-4$

4. Goldberg BS, Weston WL, Kohler PF, Harris MB, Humbert JR. Transcutaneous leukocyte migration in vivo : cellular kinetics, platelet and C5a dependent activity. I Invest Dermatol 1979; 72 : 248-52

5. Lowe BL, Van der Leun JC. Suction blis ters and dermal-epidermal adherence. $J$ Invest Dermatol 1968 ; 50 : 308-14.

6. Dubertret $\mathrm{L}$, Lebreton $\mathrm{C}$ Touraine $\mathrm{R}$ Neutrophil studies in psoriatics: in vivo migration, phagocytosis and bacterial killing. J Invest Dermatol 1982 ; 79 : 74-8.

7. Dubertret L, Lebreton C, Touraine $\mathbf{R}$. Inhibition of neutrophil migration by etretinate and its main metabolite. $\mathrm{Br} J$ Dermato $1982 ; 107: 681-5$ 
Indépendamment, une autre technique de prélèvement cutané était développée: la technique des bulles de succion [5]. L'application d'une dépression légère, de 200 à $300 \mathrm{~mm}$ de mercure, à la surface de la peau entraîne un décollement progressif et indolore de l'épiderme (figure 1-A). Le fluide interstitiel cutané vient s'accumuler entre le derme et l'épiderme et, progressivement, apparaît une bulle de succion (figure 1-B). Le décollement épidermique provoqué par la formation de la bulle se fait avec précision et reproductibilité au niveau de la jonction dermo-épidermique, à un niveau bien défini en microscopie électronique (figure 1-C). A l'origine, c'est le fluide interstitiel recueilli dans ces bulles qui intéressait les chercheurs. Le dosage de l'histamine, par exemple, a permis de mieux caractériser certaines réactions urticariennes et le dosage de la prostaglandine $\left(\mathrm{PGE}_{2}\right)$ a facilité la compréhension de la cinétique de l'inflammation associée aux coups de soleil. Ce fluide a été également utilisé en pharmacocinétique pour doser, au niveau d'un organe cible périphérique, la concentration de médicaments administrés par voie locale ou par voie générale et pour déceler la présence éventuelle de métabolites.

Il nous a paru intéressant d'associer la technique des chambres de migration et celle des bulles de succion pour pouvoir développer des études pharmacologiques quantitatives in vivo. En bref, une bulle de succion d'un demicentimètre de diamètre environ est réalisée. L'épiderme est enlevé délicatement (il peut être utilisé pour des études biochimiques et photochimiques ou pour la mise en culture de cellules épidermiques). Nous nous trouvons alors devant une fenêtre cutanée, parfaitement définie en surface et en profondeur, ouverte sur le derme et sa microvascularisation (figure 2). Cette fenêtre cutanée est recouverte par une capsule stérile en Macrolon ${ }^{\circledR}$ siliconé dans laquelle une solution peut être introduite $m / s n^{\circ} 2$ vol. 4, février 88

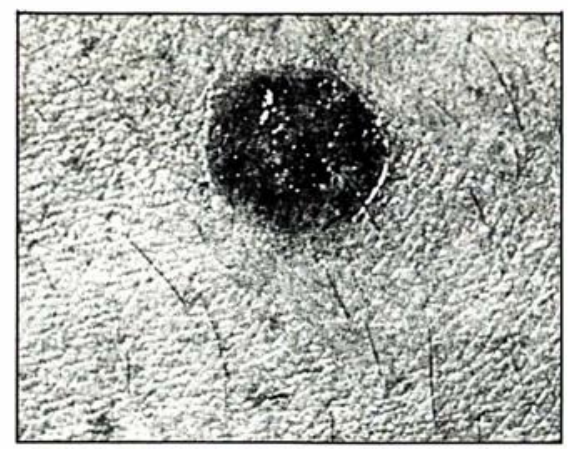

Figure 2. La fenêtre cutanée. Le fluide interstitiel accumulé dans la bulle de succion est prélevé et peut être recueilli pour différents dosages. L'épiderme qui forme le toit de la bulle est délicatement retiré avec des pinces fines stériles et peut être utilisé pour des études biochimiques variées ou pour des cultures de cellules épidermiques. Une fois l'épiderme enlevé, apparaît le derme superficiel mis à nu sans traumatisme ni douleur. La fenêtre dermique obtenue est parfaitement définie en surface et en profondeur, ce qui permet d'obtenir des résultats parfaitement reproductibles.

et renouvelée à des intervalles réguliers pendant plusieurs jours (figure 3, page 112). Une fois l'expérience terminée, la chambre est retirée. La cicatrisation se fait alors rapidement sans laisser de marque puisque le derme n'a pas été lésé.

\section{La réaction inflammatoire primaire}

Un élément commun à des réactions inflammatoires, dont la physiopathologie peut être aussi différente que les vascularites allergiques, le psoriasis ou la dermatite herpétiforme, est la migration de polynucléaires neutrophiles des capillaires vers les tissus. Ces cellules activées libèrent leurs enzymes lysosomales et leurs médiateurs lipidiques, créant d'importants dégâts tissulaires.

Afin de quantifier la réaction inflammatoire primaire et d'étudier sa modulation pharmacologique par les médicaments, nous avons introduit, dans les cham- bres de migration, du sérum autologue. Ce dernier comporte un melange de facteurs chimiotactiques connus et inconnus mais dont les plus importants semblent le complément activé ${ }^{*}$ et les produits de dégradation de la fibrine. Ces facteurs chimiotactiques sont libérés physiologiquement lors d'une blessure; ils permettent d'attirer les neutrophiles vers le site lésé, de le déterger et de lutter contre la prolifération bactérienne.

Dans un premier temps, nous avons comparé les capacités fonctionnelles (phagocytose et bactéricidie) des polynucléaires neutrophiles circulants à celles des neutrophiles ayant migré à travers la peau jusque dans la chambre [6]. Nous n'avons trouvé aucune différence chez les sujets normaux. En revanche, chez les patients psoriasiques, nous avons observé que la migration des polynucléaires neutrophiles vers le sérum autologue est très augmentée au cours des huit premières heures. Entre $8 \mathrm{~h}$ et $24 \mathrm{~h}$, au contraire, une très nette inhibition de la migration cellulaire apparaît, ce qui n'est pas observée chez les sujets normaux. La phagocytose et la bactéricidie des cellules recueillies dans la chambre sont diminuées par rapport à celles des sujets normaux. Il semble donc que les fonctions des polynucléaires neutrophiles chez les psoriasiques en poussée soient anormales. Ces cellules sont en particulier très sensibles aux stimuli chimiotactiques mais des mécanismes régulateurs s'expriment rapidement au niveau de la

\footnotetext{
* Le complément est constitué d'une vingtaine de protéines circulantes dont l'activation en cascade aboutit à la formation de composés doués d'activités biologiques diverses. En particulier, certains produits d'activation du complément peuvent provoquer ou amplifier une réaction inflammatoire locale. Les plus puissants de ces produits pro-inflammatoires sont le C3a et le C5a (anaphylatoxines). Tous deux provoquent la libération d'histamine par les mastocytes et les basophiles et, lorsqu'ils sont injectés par voie intradermique, induisent l'apparition d'un érythème et d'un cedème localisés. De plus, le C5a a un effet chimiotactique sur les polynucléaires et entraîne la libération d'enzymes lysosorniales par ces cellules.
} 
peau pour contrôler cette réaction excessive. Après traitement, lorsque le malade est blanchi, le chimiotactisme excessif des premières heures disparaît alors que l'inhibition retardée persiste. Il existe donc dans la peau des mécanismes capables non seulement de déclencher la réaction inflammatoire mais aussi de la contrôler. Cette inhibition est
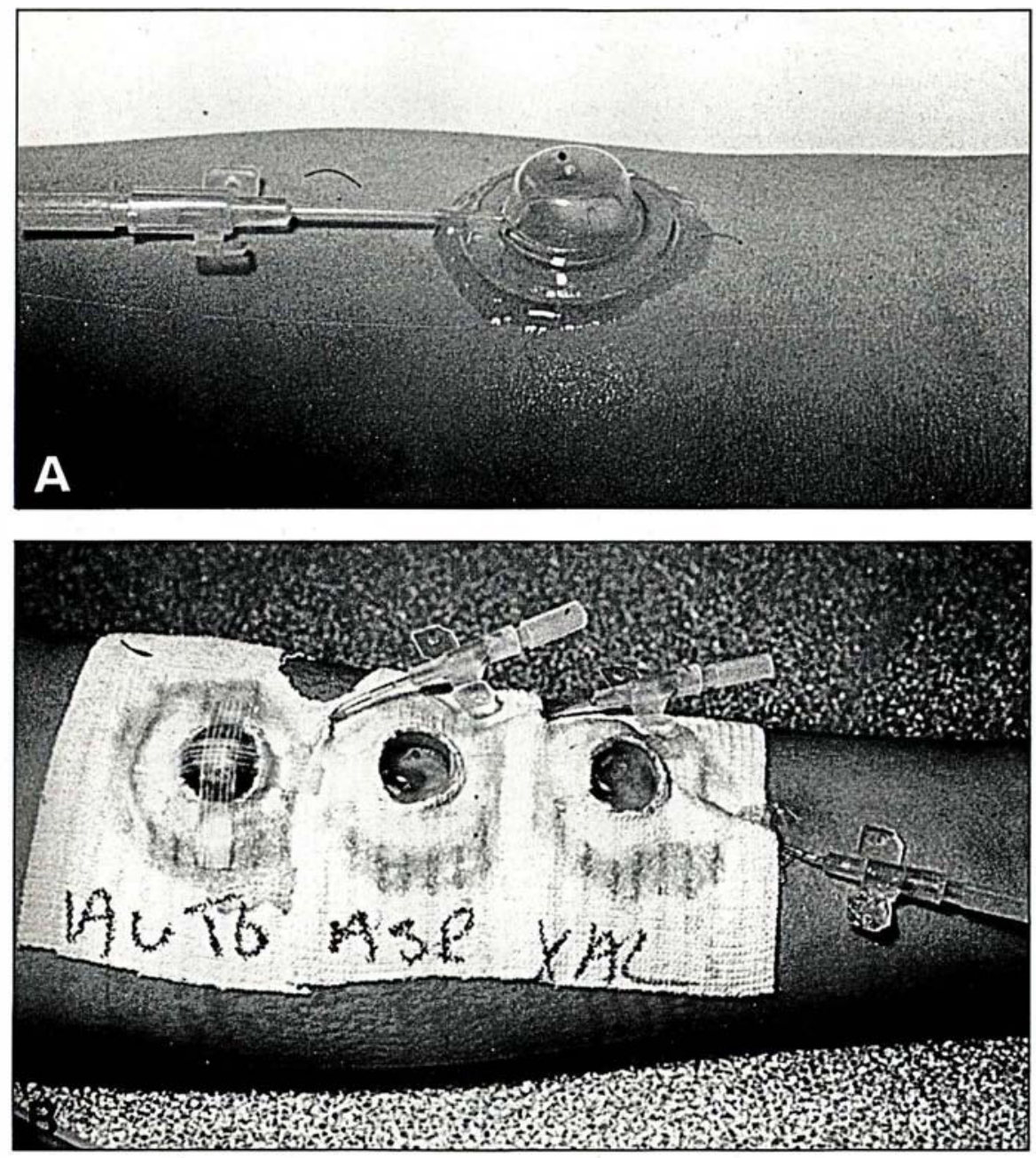

Figure 3. La chambre de migration. Une cupule stérile en Macrolon ${ }^{\circledR}$ siliconé est appliquée au-dessus d'une fenêtre dermique de $0,8 \mathrm{~mm}$ de diamètre environ et maintenue en place par de la colle et un pansement adhésif (cliché A). Cette cupule ou chambre cutanée peut rester sur l'avant-bras d'un sujet durant un temps variable allant de quelques heures à plusieurs jours. Une solution donnée $(1 \mathrm{ml})$ est introduite dans la chambre cutanée, grâce à une tubulure fine en polypropylène Vygon (囚), et peut être renouvelée à des temps précis, ce qui permet de réaliser des études cinétiques. De plus, il est possible de poser trois chambres cutanées sur l'avant-bras d'un même individu (cliché B), ce qui permet de comparer les propriétés de différentes solutions ou d'étudier, sur un même sujet, l'effet de

sans double responsable de l'autolimitation, voire de la régression observée au

De nombreuses maladies inflammatoires chroniques sont caracténucléaires neutrophiles dans les lésions. Cette accumulation provoque d'importants dégâts tissulaires. Il est donc important de plusieurs doses d'une molécule donnée. mettre au point des anti-inflammatoires sélectionnés pour leur capacité à s'opposer à la migration des neutrophiles.

Intrigués par la très grande efficacité des rétinoïdes (dérivés synthétiques de la vitamine A) dans les psoriasis inflammatoires, nous avons posé l'hypothèse que ces molécules pouvaient inhiber l'accumulation des neutrophiles [7]. Nous avons donc étudié, par la technique des chambres cutanées, la migration de ces cellules en présence de rétinoïdes dans les chambres cutanées ou avant et après administration de ces médicaments par voie systémique. Les rétinoïdes inhibent significativement la migration des cellules inflammatoires in vivo. Cependant, nous avons constaté que cette action anti-inflammatoire nécessitait des concentrations de rétinoïdes beaucoup plus élevées par voie locale que par voie systémique. Nous en avons déduit que l'action anti-inflammatoire des rétinoïdes n'est pas due uniquement à la molécule absorbée mais aussi à ses métabolites. Les recherches se poursuivent actuellement pour tenter de définir de nouvelles classes d'anti-inflammatoires qui pourraient, par des mécanismes très variés, s'opposer à la migration des polynucléaires neutrophiles des capillaires vers les tissus; de tels anti-inflammatoires devraient alors avoir des indications très larges.

\section{Action des médiateurs de I'inflammation in vivo}

De nombreux médiateurs de l'inflammation ont été identifiés au cours des dernières années et ont été caractérisés par certaines propriétés. Par exemple, le paf-acéther (platelet-activating factor) agrège les plaquettes, l'histamine augmente la perméabilité capillaire, le leucotriène $\mathrm{B}_{4}\left(\mathrm{LTB}_{4}\right)$ est un des plus puissants agents chimiotactiques connus. Le problème reste de savoir comment s'expriment ces propriétés dans la complexité de l'organe vivant et si d'autres effets insoupçonnés peuvent se manifester. Pour tenter de 


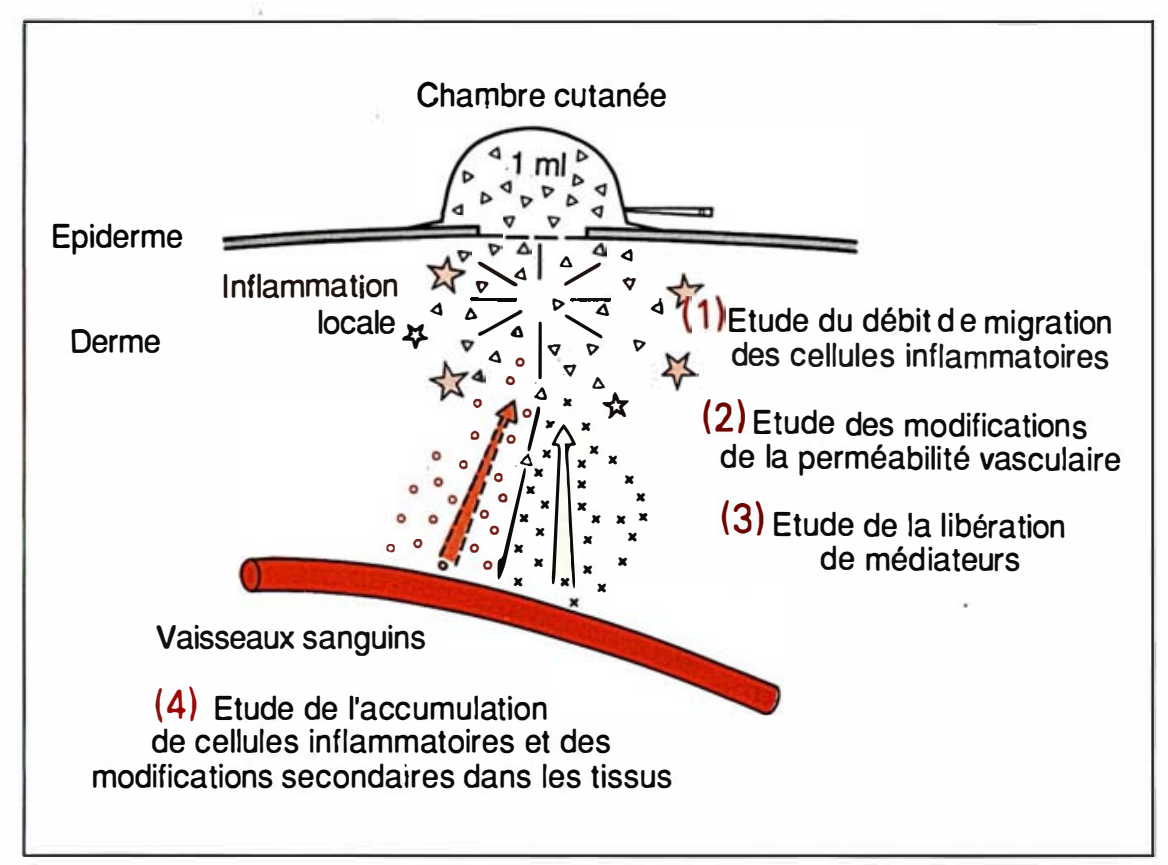

Figure 4. Schéma de la réaction inflammatoire analysée par la technique de la chambre cutanée. La chambre cutanée est appliquée sur une fenêtre ouverte sur le derme et sa microvascularisation. La solution ( $\Delta$ ) qui y est introduite diffuse progressivement selon un gradient de concentration dans le derme sousjacent ( \&). Le renouvellement de cette solution permet de maintenir de façon chronique dans le derme les molécules que l'on veut étudier et d'analyser les conséquences de ce contact prolongé. L'introduction d'une substance pro-inflammatoire dans la chambre déclenche le développement d'une réaction inflammatoire locale dont les principaux óvénements peuvent être étudiés quantitativement au cours du temps. Ainsi il esı possible d'étudier l'action d'une molécule sur: (1) le débit de migration (î) des cellules circulantes $(O)$ qui quittent les vaisseaux et pénètrent le derme jusqu'à la chambre en réponse à l'appel de la molécule (effet chémoattracteur); les différentes populations cellulaires ayant migré dans la chambre sont identifiées, comptées, et leurs capacités fonctionnelles (phagocytose et bactéricidie des polynucléaires neutrophiles par exemple) peuvent être analysées; (2) l'augmentation de la perméabilité vasculaire qui se traduit par le passage de protéines plasmatiques $(x)$ à travers les parois des vaisseaux et leur diffusion (i) dans les tissus jusqu'à la chambre où elles s'accumulent et peuvent être dosées; (3) la libération de substances pharmacologiques, comme I'histamine ou les médiateurs lipidiques, par les mastocytes du derme ou les cellules inflammatoires ayant migré dans les tissus; (4) I'accumulation de cellules inflammatoiresdans le derme et les vaisseaux, étudiée sur une biopsie de la fenêtre cutanée par des techniques de morphologie mises au point au laboratoire. L'observation en microscopie optique, et, éventuellement électronique, permet aussi d'analyser les dommages tissulaires secondaires à l'inflammation(\$)). De plus, cette technique permet de quantifier la métabolisation de la molécule étudiée et l'apparition de ses produits de dégradation dans la chambre cutanée. En utilisant le même processus expérimental; les propriétés anti-inflammatoires d'un médicament introduit dans la chambre cutanée ou administré par voie orale peuvent être déterminées. Signification des symboles. Triangles gris = solution introduite dans la chambre cutanée; cercles rouges = cellules circulantes qui migrent dans les tissus ; croix noires = protéines qui diffusent dans les tissus ; barres radiales = localisation du site de l'inflammation; étoiles roses = dommages tissulaires; flèche creuse à traits discontinus rouge $=$ débit de migration des cellules inflammatoires; flèche rose creuse à traits continus $=$ débit de diffusion des protéines; flèche noire se dirigeant vers les vaisseaux sanguins = gradient de concentration. Bas du schéma = vaisseaux sanguins en rouge ; haut du schéma (couche grise) = épiderme; traits continus noirs = diffusion dans les tissus de la molécule introduite dans la chambre cutanée.

$m / s n^{\circ} 2$ vol. 4, férier 88 répondre à ces questions, une première approche a consisté à injecter, en intradermique, un médiateur donné et à faire des biopsies séquentielles. Cependant, le traumatisme de l'aiguille, l'inactivation très rapide des médiateurs et la difficulté de localiser avec précision la zone d'injection ont quelque peu limité l'intérêt de ces expériences. Dans une réaction inflammatoire, les médiateurs sont libérés de façon chronique et diffusent selon un gradient de concentration dans les tissus. Pour mimer cette situation, nous avons utilisé la technique des chambres de migration. Le médiateur étudié est introduit dans la chambre et diffuse progressivement, sans aucun traumatisme, à travers la fenêtre cutanée. Il est alors possible de quantifier in vivo la migration des cellules inflammatoires dans la chambre de migration, d'établir quelles sont les populations cellulaires impliquées et d'analyser leur état fonctionnel (figure 4). On peut étudier les variations de la perméabilité vasculaire en mesurant la diffusion des protéines sériques dans la chambre. Le métabolisme du médiateur introduit dans la chambre peut être quantifié comme peut l'être aussi la libération d'autres médiateurs dont la sécrétion est stimulée dans le derme superficiel par la molécule étudiée. En effectuant une biopsie de la fenêtre cutanée, on peut observer, par des techniques de morphologie fine, l'infiltrat inflammatoire et les modifications tissulaires secondaires à l'inflammation. Toutes ces études peuvent être cinétiques, ce qui permet d'évaluer non seulement les processus d'activation, mais également les mécanismes d'inhibition qui modifient la réaction inflammatoire.

Nous avons pu ainsi montrer que le $\mathrm{LTB}_{4}$ est chimiotactique in vivo pour les polynucléaires neutrophiles en fonction de la dose et qu'il provoque, lorsqu'il est associé à la $\mathrm{PGE}_{2}$, une augmentation importante de la perméabilité capillaire [8]. L'histamine, intro- 
duite dans la chambre à des doses dix fois plus élevées que ces deux médiateurs, ne possède aucun effet chimiotactique pour les cellules inflammatoires mais augmente la perméabilité vasculaire dans des proportions similaires à l'association $\mathrm{LTB}_{4}$ et $\mathrm{PGE}_{2}$. La PGE seule, ne modifie ni la migration des cellules inflammatoires ni la perméabilité vasculaire. Quant au paf-acéther, il provoque l'apparition d'une diffusion intense de protéines plasmatiques hors des vaisseaux, potentialisée par l'addition de $\mathrm{PGE}_{2}$; il induit l'accumulation et la dégranulation de basophiles dans les vaisseaux sousjacents à la chambre de migration et l'infiltration de neutrophiles dans le derme superficiel.

Ces résultats mettent en évidence l'importance de la coopération synergique qui s'établit entre les différents mediateurs de l'inflammation. Ils soulignent aussi l'intérêt de la réalisation d'expériences in vivo pour étudier un médiateur donné dans un environnement tissulaire complexe où s'expriment diverses interactions, insoupçonnées in vitro. Ainsi nous avons pu observer que le $\mathrm{LTB}_{4}$ était métabolisé au contact du derme superficiel. De plus, malgré le renouvellement régulier des médiateurs étudiés dans la chambre cutanée, la migration cellulaire et la diffusion protéique reviennent à leur niveau de base au cours du temps. Ces résultats mettent en évidence l'activation de systèmes régulateurs de la réaction inflammatoire dans la peau humaine.

Nous utilisons cette méthode pour étudier les capacités de nouvelles molécules à inhiber in vivo les effets des médiateurs de l'inflammation.

\section{Hypersensibilité immédiate}

Les injections intradermiques d'allergènes sont couramment utilisées pour confirmer le diagnostic d'hypersensibilité immédiate d'un sujet au pollen, à la poussière de maison, etc. Ces antigènes, injectés dans le derme superficiel, entraînent, par un mécanisme impliquant les IgE, la dégranulation des mastocytes, entre autres, et l'apparition d'une réaction inflammatoire immédiate, suivie, dans certains cas, d'une réaction retardée. Pour mieux caractériser in vivo la réaction d'hypersensibilité immédiate et son contrôle éventuel par les médicaments, nous avons injecté du pollen dans la chambre de migration appliquée sur des sujets allergiques. Nous avons alors observé qu'en réponse au pollen apparaissent précocément une libération d'histamine et une augmentation de la perméabilité vasculaire et plus tardivement une production de $\mathrm{PGD}_{2}$ et de pafacéther ainsi qu'un recrutement intense de polynucléaires éosinophiles dans le derme. Il semble donc que la peau humaine soit un support expérimental vivant adéquat pour étudier quantitativement les événements pathologiques impliqués dans le développement de la réaction allergique et pour mettre au point de nouveaux médicaments destinés à traiter ces états d'hypersensibilité immédiate, qu'il s'agisse d'antihistaminiques ou d'inhibiteurs du paf-acéther.

En conclusion, la technique des chambres cutanées permet d'étudier de façon non agressive, quantitative et cinétique, les réactions de l'organisme à des agressions très variées. L'avantage principal de cette méthode est qu'elle permet des études pharmacologiques et pharmacocinétiques prédictives en utilisant la peau comme un organe reflet. Cependant cette approche in vivo ne diminue en rien l'intérêt des études réalisées in vitro. Bien au contraire, ces deux modes d'approche sont complémentaires et doivent être menés parallèlement, la complexité du vivant étant éclairée par des études biochimiques analytiques et de systèmes de cultures cellulaires simplifiés. De ce dialogue entre les expériences faites in vivo et in vitro devrait naître des tests prédictifs qui permettront l'étude de molécules nouvelles plus sélectives

\section{Summary}

The ability of the skin to develop inflammation, the variety of pathological conditions to which it is subject, supplemented with the fact that this organ has a large surface and is easily accessible have favored the set up of experiments on human skin. The ideal way to analyze development of inflammatory reactions is to do the actual study in vivo in man in which the main advantage is that all the various mechanisms relevant to the clinical situation are taken in account. The association of a suction blister technique with a skin chamber one provides a new experimental approach which allows to investigate pathophysiology of various inflammatory diseases as well as potential organ responses to therapeutic agents. The non invasive skin chamber technique we developped enables to quantitatively analyze the main inflammatory events occurring during an acute as well as a chronic inflammation, namely: (1) inflammatory cell migration (chemotaxis); (2) modifications in the vascular permeability (vasopermeation); (3) release of pro- and anti-inflammatory mediators by skin resident cells or/and activated infiltrated circulating cells ; (4) secondary tissue alterations. A precise molecule, such as a biological inflammatory mediator, can be introduced into the skin chamber and studied for its in vivo ability to induce or modify the events detailed above. Similarly can be studied the effects of a drug either introduced into the chamber or orally administered to normal volunteers or to patients suffering of a specific disease. Therefore, the skin chamber technique supplemented with sensitive analytical assays brings essential informations concerning skin inflammation and allows to light up some mechanisms involved in cutaneous diseases as well as to improve their pharmacological treatment. 